EPJ Web of Conferences 19, 05005 (2012)

DOI: $10.1051 /$ epjconf/20121905005

(C) Owned by the authors, published by EDP Sciences, 2012

\title{
First results for the solar neighborhood of the Asiago Red Clump Survey
}

\author{
M. Valentini ${ }^{1, a}$, U. Munari ${ }^{2}$, T. Saguner $^{2}$, K. Freeman ${ }^{3}$, S. Pasetto ${ }^{4,5}$, \\ J. Montalbán ${ }^{1}$ and E.K. Grebel ${ }^{5}$ \\ 1 Institute d'Astrophysique et de Géophysique, Université de Liége, 4000 Liège, Belgium \\ 2 INAF-OAPD-Osservatorio Astronomico di Padova, via dell'Osservatorio 8, 36012 Asiago, \\ Italy \\ ${ }^{3}$ Mount Stromlo Observatory, Australian National University, Weston Creek, ACT 2611, \\ Australia \\ ${ }^{4}$ University College London, Dept. of Space \& Climate Physics, Mullard Space Science Lab., \\ UK \\ ${ }^{5}$ Astronomisches Recheninstitut, Universitaet Heidelberg, Heidelberg, Germany
}

\begin{abstract}
The Asiago Red Clump Spectroscopic Survey (ARCS) is an ongoing survey that provides atmospheric parameters, distances and space velocities of a well selected sample of Red Clump stars distributed along the celestial equator. We used the ARCS catalog for a preliminary investigation of the Galactic disk in the Solar Neighborhood, in particular we focused on detection and characterization of moving groups.
\end{abstract}

Red Clump stars (hereafter RC stars) are a remarkable and well known tool to investigate the structure and the kinematics of various Galactic subsystems. First two data releases by Asiago Red Clump surveys (at high [7] and intermediate [5] resolving power) provide atmospheric parameters $\left(\mathrm{T}_{\text {eff }}, \log (\mathrm{g}),[\mathrm{M} / \mathrm{H}]\right)$, spectrophotometric distances and radial velocities for a well selected sample of 439 RC stars, mainly located in a torus extending from 200 to $500 \mathrm{pc}$ from the Sun (future ARCS data releases will explore both inward and outward). Table 1 shows the accuracy of the data provided by the survey.

We implemented the catalog with ages computed using the code PARAM, developed by L. Girardi [1]. The uncertainty on age is up to $40 \%-80 \%$, due to the nature of the RC: isochrones of very different age and metallicity lie closely together in this region. For this reason we used PARAM ages only for a first statistical investigation and we considered only stars with an accuracy on age better than $60 \%$. The age determination for Red Giants (and hence RC stars) will be improved by recent and exciting asteroseismology findings with data provided by CoRoT and Kepler space missions ([2-4]).

A typical analysis of the distribution of ARCS stars in the UV velocity space permits the study of the disk kinematics in the Solar Neighborhood. Different local irregularities that comes out as concentrations of stars in the U-V distribution are the moving groups or stellar streams [6, 8]. Several hypotheses are claimed to explain the origin of moving groups: dispersal stellar clusters, accretion or a resonant mechanism. A homogeneity in chemical abundances and age is expected if the moving group is the debris of star forming aggregates or of an infalling object. On the other hand, an absence of such homogeneities argues for a resonant origin (related to the Galactic bar or spiral arms).

\footnotetext{
a e-mail: valentini@astro.ulg.ac.be
}

This is an Open Access article distributed under the terms of the Creative Commons Attribution-Noncommercial License 3.0, which permits unrestricted use, distribution, and reproduction in any noncommercial medium, provided the original work is properly cited. 
Table 1. Accuracy of the ARCS high and medium resolution surveys.

\begin{tabular}{lllll}
\hline & $\mathrm{V}_{\text {rad }}$ & $\mathrm{T}_{\text {eff }}$ & $\log (\mathrm{g})$ & {$[\mathrm{M} / \mathrm{H}]$} \\
\hline ARCS high & $0.5 \mathrm{~km} \mathrm{~s}^{-1}$ & $55 \mathrm{~K}$ & $0.12 \mathrm{dex}$ & $0.11 \mathrm{dex}$ \\
ARCS medium & $1.3 \mathrm{~km} \mathrm{~s}^{-1}$ & $88 \mathrm{~K}$ & $0.38 \mathrm{dex}$ & $0.17 \mathrm{dex}$ \\
\hline
\end{tabular}

Table 2. Characteristic $\mathrm{U}$ and $\mathrm{V}$ velocities, metallicity and age of most prominent features detected in the U-V distribution of ARCS Red Clump stars. Results obtained with ARCS catalog are compared with those present in the catalog of moving groups of Zhao [8].

\begin{tabular}{|c|c|c|c|c|c|}
\hline \multirow[t]{3}{*}{ ID } & \multicolumn{2}{|l|}{ ARCS } & \multicolumn{3}{|c|}{ Zhao et al. (2009) } \\
\hline & $(\mathrm{U}, \mathrm{V})$ & {$[\mathrm{M} / \mathrm{H}]$} & Age & $(\mathrm{U}, \mathrm{V})$ & {$[\mathrm{M} / \mathrm{H}]$} \\
\hline & $\mathrm{km} \mathrm{s}^{-1}$ & $\operatorname{dex}$ & Gyr & $\mathrm{km} \mathrm{s}^{-1}$ & $\operatorname{dex}$ \\
\hline Sirius-UMa & $(+0,+7)$ & $-0.11 \sigma=0.22$ & $1.8 \sigma=3.0$ & $(+10,-14)$ & $-0.21 \sigma=0.15$ \\
\hline Coma & $(-11,-20)$ & $-0.12 \sigma=0.20$ & $1.5 \sigma=3.0$ & $(-38,-17)$ & $-0.09 \sigma=0.17$ \\
\hline Pleiades & $(-8,-22)$ & $-0.18 \sigma=0.26$ & $3.0 \sigma=1.8$ & $(-15,-23)$ & $-0.17 \sigma=0.17$ \\
\hline Hercules & $(-10,-40)$ & $-0.10 \sigma=0.29$ & $5.0 \sigma=3.0$ & $(-35,-51)$ & $-0.16 \sigma=0.20$ \\
\hline
\end{tabular}

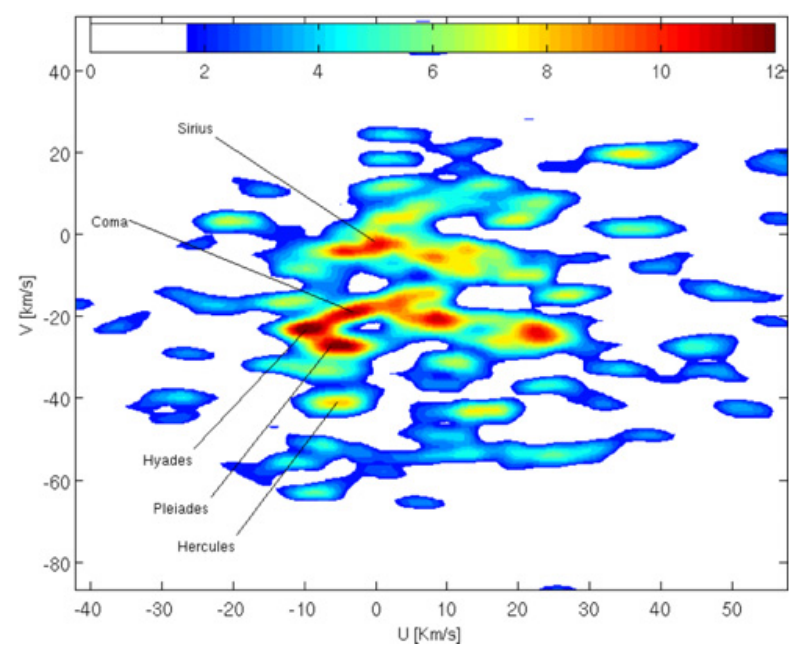

Figure 1. 2-D wavelet transform with a Mexican-hat kernel of the U-V distribution of ARCS Red Clump stars. The scale parameter of the analyzing wavelet is $5 \mathrm{~km} \mathrm{~s}^{-1}$, the color scale is adjusted in order to emphasize overdensities.

A 2-D wavelet transform technique is used to identify moving groups in the U-V plane, as performed by Skuljan [6]. We used a Mexican-hat-shaped kernel function with a scale parameter of $5 \mathrm{~km} \mathrm{~s}^{-1}$ (see Figure 1). Most known moving groups are clearly visible in the ARCS sample. The high accuracy of the data provided by ARCS exclude that detected over-densities are artifacts. In Table 1 we summarized the characteristics of the most prominent moving groups and we compared our results with those of Zhao [8]. There is good agreement between the two works and discrepancies between $(\mathrm{U}, \mathrm{V})$ coordinates of the moving groups are mainly due to a different $\mathrm{U}_{\odot} \mathrm{V}_{\odot} \mathrm{W}_{\odot}$ adopted. The large dispersion in metallicity and age argues in favour of a resonant origin of the moving groups of Sirius-UMa, Coma, Hyades, Pleiades and Hercules. In addiction to these moving groups, Fig. 1 shows other overdensities that are discussed in detail elsewhere (Valentini et al. 2011, submitted). 
Assembling the Puzzle of the Milky Way

\section{References}

[1] da Silva L., et al., A\&A 458, (2006) 609

[2] Miglio A., et al. (2011), "Assembling the Puzzle of the Milky Way" proceedings

[3] Montalbán J. et al., ApJL 721, (2010) 182

[4] Mosser, B. et al., A\&A 517, (2010) 22

[5] Saguner T., Munari U., Fiorucci M., Vallenari A., A\&A 527, (2011) A40

[6] Skuljan J., Hearnshaw J. B., Cottrell P. L., MNRAS 308, (1999) 731

[7] Valentini M., Munari U., A\&A 522, (2010) A79

[8] Zhao J., Zhao G., Chen Y., ApJ 692, (2009) L113 